Korean J Ophthalmol 2021;35(3):::248-250 https://doi.org/10.3341/kjo.2020.1124

\section{Macular Hole Formation Following Vitrectomy for Ruptured Retinal Arterial Macroaneurysm}

\section{Dear Editor,}

Retinal arterial macroaneurysm (RAM) is an acquired, focal dilation of the retinal artery. Hemorrhages from a macroaneurysm can be located in the vitreous cavity and any of the retinal layers: preretinal hemorrhages, sub-internal limiting membrane (sub-ILM) hemorrhages, intraretinal hemorrhages, and subretinal hemorrhages. A ruptured RAM may rarely leads to macular hole $(\mathrm{MH})$ formation [13]. $\mathrm{MH}$ formation secondary to a ruptured macroaneurysm may occur due to mechanical damage caused by expulsion of blood from the vessels, and the distance of the RAM from the fovea is known to be strongly related to $\mathrm{MH}$ occurrence [1,2]. No previous studies on the occurrence of $\mathrm{MH}$ after pars plana vitrectomy (PPV) for a ruptured RAM have been reported. We report a case of MH formation after vitrectomy and describe the mechanism of $\mathrm{MH}$ formation associated with RAM rupture.

A 72-year-old female with a history of diabetes mellitus and uncontrolled hypertension, visited our hospital because of visual deterioration in the left eye for 3 days without previous trauma or eye surgery. The best-corrected visual acuity of the left eye was finger count $10 \mathrm{~cm}$, and the intraocular pressure was $11 \mathrm{mmHg}$ by Goldman applanation tonometry. Fundus examination revealed a ruptured RAM at the superior branch of the retinal artery and an approximately 1.2 disc diameter-sized multilevel hemorrhage (Fig. 1A). Optical coherence tomography (OCT; Optovue, Fremont, CA, USA) showed thick sub-ILM and subretinal hemorrhages involving the macula from the ruptured macroaneurysm (Fig. 1B). The central macular thickness was $509 \mu \mathrm{m}$, and the inner retinal thickness was $102 \mu \mathrm{m}$.

Received: December 9, 2020 Final revision: April 13, 2021

Accepted: April 21, 2021
We performed a 25-gauge three-port PPV and ILM peeling using end-grips forceps. We removed a thick blood clot from under the ILM using a soft-tip backflush instrument and displacement of the subretinal hemorrhage by instilling $22 \%$ sulfur hexafluoride gas tamponade through fluidgas exchange. The patient remained in the prone position for 1 week postoperatively. During the postoperative period, the subretinal hemorrhage gradually decreased and migrated to the peripheral area (Fig. 1C). One month later, the left eye cataract was aggravated, so phacoemulsification and posterior chamber intraocular lens implantation were performed without any complications.

Subretinal blood clots remained and the macular thickness decreased 2 weeks after cataract surgery (Fig. 1D). Eight weeks after PPV, the subretinal hemorrhage in the macular area was completely absorbed, and a full-thickness MH was detected via OCT about $282 \mu \mathrm{m}$ in minimal diameter (Fig. 1E). The patient declined further treatment, therefore, additional intravitreal gas injection and surgery were not performed. Five months postoperatively, the best-corrected visual acuity of the left eye was finger count $10 \mathrm{~cm}$ with no change and the hole was still visible on fundus examination (Fig. 1F).

Several mechanisms for $\mathrm{MH}$ formation associated with a ruptured RAM can be explained. First, MH and abrupt RAM rupture can occur consecutively or simultaneously. Mechanical damage caused by expulsion of blood from the vessels can cause MH formation [1-3]. Second, sub-ILM hemorrhage, preretinal hemorrhage, vitreous hemorrhage after a RAM rupture can cause alteration of anterior-posterior vitreal traction, thus contributing to $\mathrm{MH}$ formation [4]. Third, iatrogenic traction forces during vitrectomy and ILM peeling can induce intraoperative $\mathrm{MH}$ formation [3]. Fourth, sustained subretinal hemorrhage can contribute to $\mathrm{MH}$ formation from retinal retraction due to fibrin derived from blood clots [5].

Our case identified MH formation after PPV in ruptured RAM using OCT serial image. We removed sub-ILM hemorrhage by surgery, but the subretinal hemorrhage was still sustained in the subfoveal area for more than 1 month, the macular thickness gradually decreased, and eventually, the hole was formed. There was no abnormal epiretinal membrane postoperatively. We suggest that $\mathrm{MH}$ formation 

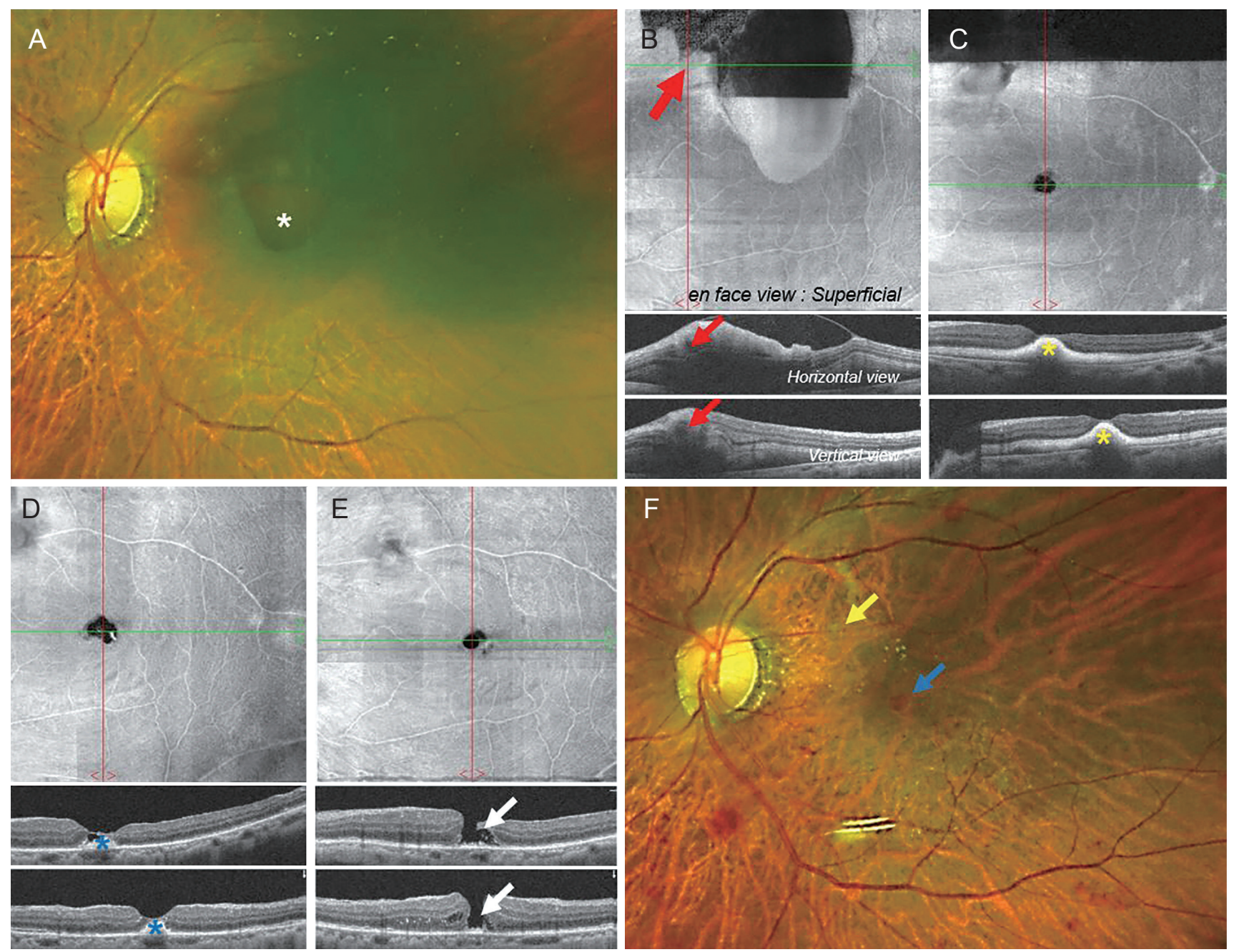

Fig. 1. Wide-field fundus photography (WF) images and spectral-domain optical coherence tomography (OCT) images during the macular hole formation : upper panel : en face view, lower panel : B-scan view. (A) At first visit, preoperative WF image shows the multileveled retinal hemorrhage (asterisk) and a retinal arterial macroaneurysm (RAM) at the superior branch of the retinal artery. (B) At first visit, preoperative OCT image shows the subinternal limiting membrane hemorrhage and the subretinal hemorrhage from a ruptured RAM (arrows). (C) At 1 week postvitrectomy, the OCT image shows a remained subretinal hemorrhages (asterisks). (D) One month postvitrectomy, the OCT image shows a thinned retina with blood clots in subretinal space (asterisks). (E) Two months postvitrectomy, OCT image shows a full-thickness macular hole (arrows). There is no epiretinal membrane above the hole. (F) Five months postvitrectomy, WF image shows a RAM at the superotemporal branch (yellow arrow) and a macular hole (blue arrow).

related to a long-lasting subretinal hemorrhage in the macular area that led to dehiscence of the fovea [3,5]. Additionally macrophage's clearance progress for organized blood clots could play a part by its over-action.

In conclusion, we report an uncommon case of MH formation associated with a ruptured RAM. The presence of long-lasting subretinal hemorrhage may contribute to $\mathrm{MH}$ formation after vitrectomy and ILM peeling in patients with RAM.
Jae Hong An, Ji Hye Jang

Department of Ophthalmology, Keimyung University School of Medicine, Daegu, Korea

E-mail (Ji Hye Jang):mjmom99@dsmc.or.kr

\section{Conflict of Interest}

No potential conflict of interest relevant to this article was reported. 


\section{References}

1. Tashimo A, Mitamura Y, Ohtsuka K, et al. Macular hole formation following ruptured retinal arterial macroaneurysm. Am J Ophthalmol 2003;135:487-92.

2. Sato R, Yasukawa T, Hirano Y, Ogura Y. Early-onset macular holes following ruptured retinal arterial macroaneurysms. Graefes Arch Clin Exp Ophthalmol 2008;246:177982.
3. Sagara N, Kawaji T, Koshiyama Y, et al. Macular hole formation after macular haemorrhage associated with rupture of retinal arterial macroaneurysm. Br J Ophthalmol 2009; 93:1337-40.

4. Lumi X, Drnovsek F. Management of a macular hole following retinal arterial macroaneurysm rupture. Am J Case Rep 2020;21:e922437.

5. Colucciello M, Nachbar JG. Macular hole following ruptured retinal arterial macroaneurysm. Retina 2000;20:94-6. 